\title{
System deployment and decentralized control of islanded AC microgrids without communication facility
}

\author{
Baoquan $\operatorname{LIU}^{1}$ (i)
}

\begin{abstract}
This paper proposes a novel system deployment principle for master/slave type islanded alternating current (AC) microgrids, with which decentralized control can be achieved without communications. The net power of a microgrid, including active and reactive power, is metered and compensated locally and independently by its units. This can benefit a microgrid regarding system expandability, flexibility, and plug-and-play. The proposed strategy is demonstrated in a typical islanded AC microgrid with diesel generators, renewable generation, and hybrid storage. A diesel generator set with constant speed governor and static exciter runs to build up and dominate the main AC bus. An ultra-capacitor unit suppresses fastvarying power fluctuations, and the battery shares part of the slow-varying power component. The diesel generator set only provides slow-varying power within a lower limit, which can avoid dramatic accelerations and decelerations and low load-rate operation. Finally, simulations on MATLAB/Simulink are carried out to verify the proposed strategy in typical scenarios.
\end{abstract}

Keywords System deployment, Decentralized control, AC microgrid, Diesel, Hybrid storage

CrossCheck date: 20 September 2018

Received: 2 May 2018/Accepted: 20 September 2018/Published online: 20 November 2018

(C) The Author(s) 2018

$\triangle$ Baoquan LIU

liubq@sust.edu.cn

1 School of Electrical and Information Engineering, Shaanxi University of Science and Technology, Xi' an 710021, China

\section{Introduction}

Along with the accelerated development of distributed generation (DG), such as photovoltaic solar arrays, wind turbines, etc., the microgrid has gradually become a promising framework for distributed energy harvesting and utilization, especially in rural and remote areas that a utility grid cannot reach [1-3]. A microgrid is typically a low voltage network consisting of DGs, local loads, energy storage, and other auxiliary infrastructure, aiming to power a certain area (e.g., an island, an industrial park, or a residential quarter) [4-6].

To achieve power balance and further economical operational objectives, centralized to distributed control frameworks can be applied [7, 8]. Centralized control requires an advanced central controller, which is responsible for achieving common goal(s) of the system. Through communication facilities, the central controller manages all units by collecting information, making decisions, and sending instructions [9, 10]. Control is always implemented hierarchically with two or three layers. The microgrid in [11] applies a master/slave architecture, where a master converter is assigned to dominate the $\mathrm{AC}$ bus and also performs as the central controller to drive other slave converters. Droop-based control is another system for organizing microgrid, which enables active and reactive power sharing without communications. However, the system frequency and voltage in steady state deviate from their nominal values with load changes, which must be restored using a communication-based secondary control loop [12-14]. In [15], two secondary control schemes, a model predictive controller and a Smith predictor-based controller, are investigated. In [16], two control techniques, based on $H_{\infty}$ and $\mu$-synthesis theory, are developed as the secondary correction loop. 
Distributed control has no central controller, and the information-collecting and decision-making authority is delegated to DGs and flexible loads, forming a multi-agent control architecture. In this case, DGs can operate with more autonomy, but communications, especially between adjacent units, are still necessary for accurate power sharing, frequency restoration, or to achieve economic targets [17, 18]. Reference [19] proposes a supervisory control scheme based on neighboring message exchange, which can achieve either precise power sharing or economic dispatch of a microgrid. In [20], construction rules for communication networks and their agents in a microgrid are proposed together with a systematic method to design control laws, which is verified in the study.

Generally, energy management of a microgrid with centralized or distributed control always relies on wired or wireless communications to achieve power balance, frequency restoration, or to achieve profitable targets, which will be costly and prone disturbances when it attempts to contact every unit in a complex, scattered, and unorganized system. If faults occur in the communication channels (for example, total net power information errors), the microgrid will have difficulties balancing the power within this system, likely leading to system collapse. Furthermore, the communication network, which has a particular structure and specific protocols, is relatively exclusive and not flexible for plug-and-play. Reducing the communication dependency in microgrid operations is always a challenging problem.

This work proposes a novel system deployment principle for master/slave type islanded alternating current (AC) microgrids, with which fully decentralized control without communications can be achieved. The total net power, including active and reactive power, can be metered and compensated for locally and independently by the microgrid units. This design can benefit a microgrid regarding system expandability, flexibility, and plug-and-play. A typical small-scale AC microgrid is considered for demonstration. The assumed units include a diesel generator set, renewable generation, critical loads, hybrid energy storage (battery and ultra-capacitor), and flexible loads. The diesel generator set runs to build up and dominate the $\mathrm{AC}$ bus, assuring constant frequency and voltage. With the deployment principle and the designed power flow control scheme, net power of the microgrid is separated into fastvarying and slow-varying components, which are then shared and compensated independently and locally.

The rest of this work is organized as follows. Section 2 presents the proposed system deployment principle in a typical master/slave type islanded AC microgrid. Section 3 illustrates the decentralized power flow control scheme without communications, and Section 4 provides the simulation results in typical scenarios using the
MATLAB/Simulink platform. Finally, Section 5 presents the conclusions.

\section{System deployment principle of islanded AC microgrids}

\subsection{Units of microgrid}

Up to now, diesel generators remain the de-facto power supplies for emergency and remote area power utilization with proven reliability [21, 22]. A typical islanded AC microgrid is constructed in this work using a diesel generator set with constant speed governor and static exciter to act as the grid-forming unit (master unit). Hybrid energy storage and renewable generation (wind turbines in this work) operate in the grid-feeding mode (current control as the slave unit). Furthermore, the wind turbine works in the maximum power point tracking (MPPT) mode to harvest energy.

As a rotating machine set, the diesel generator set has the following characteristics:

1) Dynamic response takes seconds or even longer, and it cannot follow the volatile renewable power fluctuations in a microgrid. Furthermore, frequent accelerations and decelerations will increase the risk of engine failures.

2) Low load-rate running (below $30 \%$ of the rated capacity) generates excessive exhaust gases due to incomplete combustion of fuel. Low efficiency also leads to premature aging of the engine owing to carbon deposits and liner glazing.

To compensate for the volatile power variations from DGs and local loads, energy storage is always essential. According to the theory applied to Ragone plots, any single type of storage device with limited capacity is not wellqualified [23]. A battery has large energy density but limited power rating and further has a short life cycle. In contrast, an ultra-capacitor can absorb/deliver large power in a short time and has a relatively long-life cycle. Their combination forms a hybrid energy storage system, providing a feasible solution in power compensation that performs effectively over an extended lifetime [24, 25].

To avoid low load-rate operation of a diesel engine, minimum power output is limited to $30 \%-40 \%$ of its rated capacity. Consequently, a flexible load is equipped as a companion to dump the surplus power when renewable generation is at a high production rate rather than reducing the diesel engine output below $30 \%$. Flexible loads can be heat production, hydrogen production, or sea water desalination systems, for example, and the type of load used depends on local conditions. To illustrate the main 
significance of the deployment principle and power flow control scheme, this work will not detail each dumping technology and preferentially takes a power converter interfaced nonlinear load as a general flexible load.

\subsection{System deployment principles}

This work proposes three system deployment principles for master/slave type islanded AC microgrids.

1) Deploy the diesel generator set together with flexible loads on one side of the main AC bus, working as the grid-forming unit.

The diesel generator set works as the grid-forming unit to build up and dominate the main $\mathrm{AC}$ bus. It connects to one terminal together with its flexible loads and downstream sub-feeders are used to connect the DGs and loads. A typical microgrid skeleton is the result.

2) Sub-feeders are used to connect renewable sources and loads. They are deployed on the opposite side of the main $\mathrm{AC}$ bus.

The total power production from renewable sources is defined as $P_{\text {gen }}$ and the total load (except for the flexible load) is defined as $P_{\text {load }}$. Then the total net power of the microgrid can be expressed as:

$P_{\text {net }}=P_{\text {gen }}-P_{\text {load }}$

To achieve power balance, $P_{\text {net }}$ should be calculated in real-time by collecting information from DGs and loads. In a conventional scattered and unorganized complex AC microgrid, a communications facility is indispensable.

In contrast, when clusters of renewable generation sources and local loads are connected together, renewable generation $P_{g e n}$ is set preferentially to satisfy the local loads represented by $P_{\text {load }}$, and they will then perform as a single entity. If this entity is interfaced to the AC bus through a single point, the surplus power $P_{\text {net }}$ will be delivered directly to the AC bus through this junction. $P_{\text {net }}$ can thus be calculated simply by metering the voltages and currents of this single point. With this deployment principle, the $P_{\text {net }}$ calculation does not rely on any communications.

\section{3) Deploy the hybrid energy storage.}

Hybrid storage is deployed between the two systems (diesel generators and renewable sources) on the main AC bus. Through power sharing and compensation, the hybrid storage system can balance the net power of the microgrid and also maintains high efficiency in the diesel engine set.

\subsection{Typical islanded AC microgrid layout}

With the above three deployment principles, the configuration of a typical master/slave type islanded AC microgrid is schematically displayed in Fig. 1. A diesel generator set connects to the left side of the main $\mathrm{AC}$ bus with its flexible load nearby. Feeders of wind turbines, $R$ and $L$ loads, and converter loads are arranged on the right side of the AC bus as a single entity, which connects to the $\mathrm{AC}$ bus through a single point. Then battery and ultracapacitor units, interfaced to the main $\mathrm{AC}$ bus through $\mathrm{DC} /$ AC converters, are located in between. The line impedances are defined as $Z_{1}, Z_{2}, Z_{3}, Z_{4}$, and $Z_{5}$.

As depicted in Fig. 1, five typical power components with associated directions, $P_{n e t}, P_{c a p}, P_{b a t}, P_{f l e x}$, and $P_{d e}$, are defined. $P_{n e t}$ is the power gap between $P_{\text {gen }}$ and $P_{\text {load }}$, and is also the active net power of the microgrid. $P_{c a p}$ and $P_{b a t}$ are defined as the power shares (portions) of the ultra-capacitor and the battery. $P_{\text {flex }}$ is the power dissipation of the flexible load and $P_{d e}$ is the power production of the diesel generator set. In addition, the minimum power production limit of the diesel engine is defined as $P_{D E}$, which is within $30 \%-40 \%$ of its nameplate capacity.

For a fully decentralized control framework, the ultracapacitor, the battery, and the flexible load only need local measurement and control loops to achieve power balance without communications. As displayed in Fig. 1, the local metering points of the three units are $A, B$, and $C$, respectively. The three-phase voltages and currents of point $A\left(\boldsymbol{v}_{A}, \boldsymbol{i}_{A}\right)$ are detected by the ultra-capacitor unit for local power calculation. Similarly, $\left(\boldsymbol{v}_{B}, \boldsymbol{i}_{B}\right)$ and $\left(\boldsymbol{v}_{C}, \boldsymbol{i}_{C}\right)$ at $B$ and $C$ are respectively metered by the battery and flexible load unit independently.

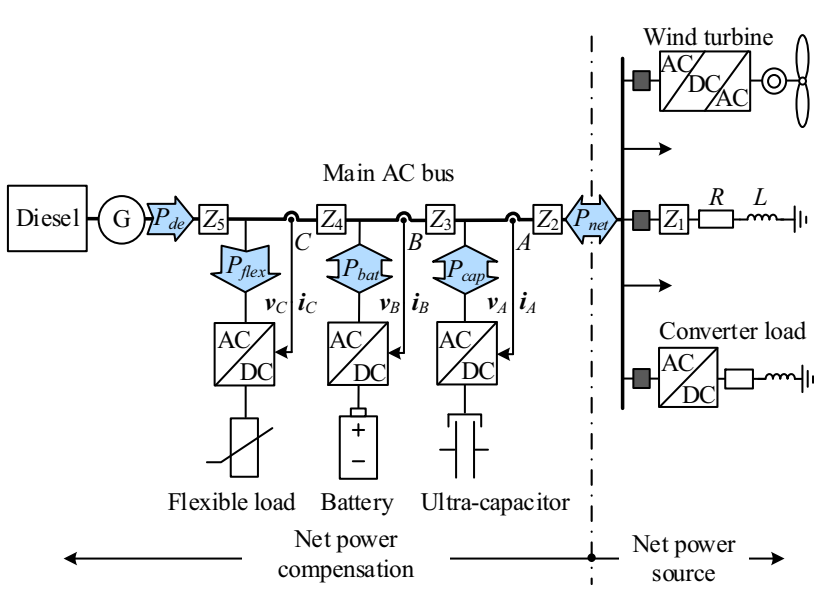

Fig. 1 Deployment of typical islanded AC microgrid 


\section{Decentralized control scheme of islanded AC microgrid}

\subsection{Local power calculation}

To derive the power shares of each unit through metered voltages and currents in a three-phase AC microgrid, phase angles of the grid voltages $\varphi$ should first be estimated through a phased-lock-loop (PLL) algorithm. As displayed in Fig. 2, $\left(v_{a}, v_{b}, v_{c}\right)$ are transformed to $v_{d}$ and $v_{q}$ under a synchronous rotating reference frame $(d-q-0)$ with transformation matrix $\boldsymbol{T}_{a b c / d q 0}$ in (2). $\omega_{f}$ is the nominal value of the grid frequency, which is a constant, and $\omega_{a}$ is the estimated frequency. By designing a feedback control loop, $v_{q}$ is regulated to zero and phase angle $\varphi$ is finally captured.

$\boldsymbol{T}_{a b c / d q 0}=\frac{2}{3}\left[\begin{array}{lll}\cos (\varphi) & \cos \left(\varphi-\frac{2 \pi}{3}\right) & \cos \left(\varphi+\frac{2 \pi}{3}\right) \\ -\sin (\varphi) & -\sin \left(\varphi-\frac{2 \pi}{3}\right) & -\sin \left(\varphi+\frac{2 \pi}{3}\right) \\ \frac{1}{2} & \frac{1}{2} & \frac{1}{2}\end{array}\right]$

With known estimated phase angle $\varphi$ and $\boldsymbol{T}_{a b c / d q 0}$, currents $\left(i_{a}, i_{b}, i_{c}\right)$ are transformed to $i_{d}$ and $i_{q}$, and then active and reactive power can be calculated through (3) and (4). This power calculation process is displayed in Fig. 3 below.

$P=\frac{3}{2}\left(v_{d} i_{d}+v_{q} i_{q}\right)$

$Q=\frac{3}{2}\left(v_{q} i_{d}-v_{d} i_{q}\right)$

\subsection{Local control of ultra-capacitor unit}

In the decentralized control framework, power detecting and sharing of all units are implemented locally and independently without communications. For the ultra-capacitor unit, as displayed in Fig. 1, voltage $\boldsymbol{v}_{A}$ and current $\boldsymbol{i}_{A}$ of point $A$ are metered locally by the voltage source converter (VSC) controller. The active and reactive power calculated by (3) and (4) are exactly equal to the net power $P_{\text {net }}$ and $Q_{\text {net }}$.

Ultra-capacitors have a large power density and longlife cycle, and can be charged or discharged even with

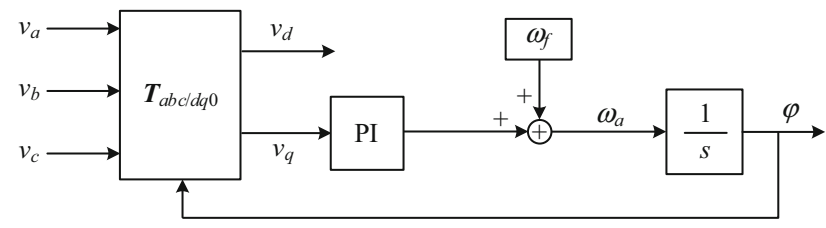

Fig. 2 PLL algorithm in three-phase AC microgrid

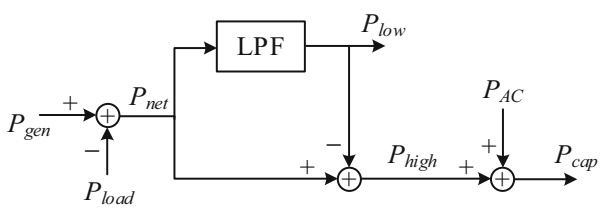

Fig. 3 Local power calculations and shares of ultra-capacitor unit

large current. The hybrid system is used to suppress the fast-varying components of $P_{n e t}$ and $Q_{n e t}$. The power sharing process is presented in Fig. $3, P_{\text {net }}$ is separated into high and low frequency components $\left(P_{\text {high }}\right.$ and $\left.P_{\text {low }}\right)$ through a low pass filter (LPF). $Q_{n e t}$ also applies the same technique to derive $Q_{\text {high }}$ and $Q_{\text {low }}$. The transfer function of the LPF is $G(s)=1 /(1+\tau s)$ and its bandwidth is $\omega_{b}=2 \pi /$ $T=1 / \tau$. The time constant $\tau$ determines the segmentation of $P_{\text {low }}$ and $P_{\text {high }}$. This work sets $\tau=30 / \pi$ so that the period $T$ at $\omega_{b}$ equals $60 \mathrm{~s}$. This indicates that power fluctuations whose varying periods are within $60 \mathrm{~s}$ will be extracted.

$Q_{h i g h}$ is then translated to $i_{q C}^{*}$ and is directly fed to the VSC controller as the reactive current reference. In contrast, $P_{\text {high }}$ is added with another power component $P_{A C}$, and their sum is the final $P_{c a p}$, which is translated to $i_{d C}^{*}$ as the active current reference.

The additional power term $P_{A C}$ is designed to rebalance the ultra-capacitor. As aforementioned, the ultra-capacitor is used to suppress any fast-varying $P_{h i g h}$. Owing to irregular power variation and self-discharge operations, its terminal voltage $v_{\text {cap }}$ can become out of balance over extended operation. Therefore, a rebalance approach is developed. The main point is to define a tolerable voltage range (no rebalance is needed in this range) with an upper limit $V_{u p}$ and a lower limit $V_{\text {low }}$, and adjust the power flowing in and out to regulate its terminal voltage.

The exact curve of $P_{A C}$ is shown in Fig. 4, and it displays a hysteretic character. If the ultra-capacitor voltage is equal to the nominal value $V_{\text {rated }}, P_{A C}$ is zero, whereas if its voltage goes beyond either limit, a corresponding $P_{A C}$ proportional to the voltage deviation is generated for voltage restoration until the ultra-capacitor's voltage returns to within the defined range. Then $P_{A C}$ becomes

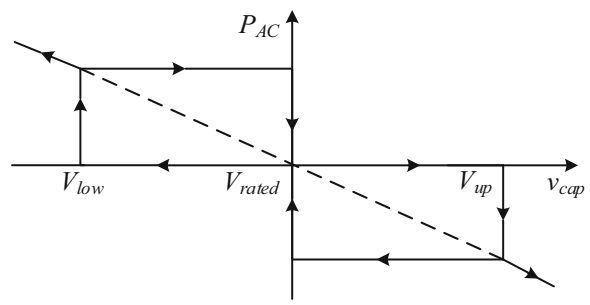

Fig. $4 P_{A C}$ used to rebalance ultra-capacitor 
constant until the voltage reaches $V_{\text {rated }}$, at which time $P_{A C}$ returns to zero.

\subsection{Local control of battery unit}

With local metering and control, the fast-varying components in $P_{n e t}$ and $Q_{n e t}$ are extracted and suppressed by the ultra-capacitor unit independently. The slow-varying components $P_{\text {low }}$ and $Q_{\text {low }}$ remain to be considered for further compensation. As deployed in Fig. 1, the battery unit is located behind (downstream of) the ultra-capacitor unit and by metering voltage $\boldsymbol{v}_{B}$ and current $\boldsymbol{i}_{B}$ at point $B$, $P_{\text {low }}$ and $Q_{\text {low }}$ can be calculated locally by the VSC of the battery. Then the battery unit endeavors to share the slowvarying power based on its storage and output capacity.

Generally, a battery's maximum charge current varies with its state of charge (SOC) and its discharge has limited power rating. In this work, a three-stage approach is followed for battery charging without SOC estimations. As shown in Fig. 5a, stage 1 involves constant current $I_{C H A}$ with limited voltage $V_{C H A}$ until the battery's voltage rises to $V_{C H A}$. Then stage 2 follows, which is a constant voltage $\left(V_{C H A}\right)$ process with the charge current decreased to a trickle value. At this time, the battery is considered to be fully charged and enters stage 3 . Through setting a smaller voltage set-point $V_{F}$, the full state of the battery is maintained in this stage. As to the discharge process, it depends on system demands, which should not exceed the discharge limit $I_{D I S}$.

The referred rules are integrated into the battery power sharing process, and referring to the schematic in Fig. 6, two profiles, $P_{\text {low }}+P_{D E}$ and $P_{b a t}$, are included. It should be noted that the diesel generator is forced to output $P_{D E}$ even without load demands, and it is preferable to charge

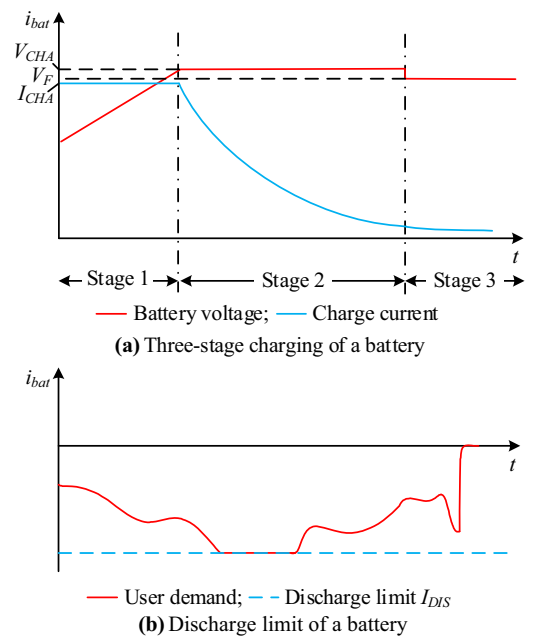

Fig. 5 Charging and discharging of a battery the battery rather than dumping its charge. Therefore, the battery takes power $P_{\text {cap }}$ from $P_{\text {low }}+P_{D E}$. If $P_{\text {low }}+P_{D E}>0$, the battery absorbs power segment $\mathrm{C}$ according to its present capability. If $P_{\text {low }}+P_{D E}<0$, the battery is discharged to compensate for power deficit D without exceeding its discharge limit $P_{D I S}$. This diagram demonstrates how to extract $P_{\text {bat }}$ from $P_{\text {low }}+P_{D E}$ and is achieved through local control of the battery VSC.

The battery VSC works as a rectifier and the control scheme is schematically displayed in Fig. 7, where an inner current loop and an outer voltage loop are designed with a saturation block between them.

The voltage loop changes set-points $V_{C H A}$ and $V_{F}$ to switch between charging stages 2 and 3 . A controller $\left(\mathrm{PI}_{3}\right)$ with an upper limit $I_{C H A}$ and a lower limit $I_{D I S}$ is designed. Its output $i_{B}^{*}$ is assumed to be the reference charging and discharging current of the battery, whereas in the current control loop, feedback $i_{d B}$ is the inductor current of the VSC under a $d$ - $q$ - 0 frame. Therefore, battery current $i_{B}^{*}$ is first converted to VSC current $i_{B}^{* *}$ through a coefficient $m$, and is then input to a dynamic saturation block. $m$ is given by (5), where $v_{\text {bat }}$ is the terminal voltage of the battery and $v_{d B}$ is the voltage of point $B$ under the $d-q-0$ frame.

$m=\frac{2}{3} \frac{v_{b a t}}{v_{d B}}$

The dynamic saturation block has a constant lower limit $I_{d D I S}$ and a dynamic upper limit $i_{\text {low }}$, given by (6) and (7), respectively.

$I_{d D I S}=\frac{2}{3} I_{D I S} \frac{v_{b a t}}{v_{d B}}$

$i_{\text {low }}=\frac{2}{3} \frac{P_{\text {low }}+P_{D E}}{v_{d B}}$

If $P_{\text {low }}+P_{D E}>0$ and the battery stays in stage 1 of the charge process, then $V_{C H A}>v_{\text {bat }}$ is always satisfied and $\mathrm{PI}_{3}$ outputs its maximum value of $I_{C H A}$. Through the saturation block, the current reference $i_{d B}^{*}$ is derived as (8).

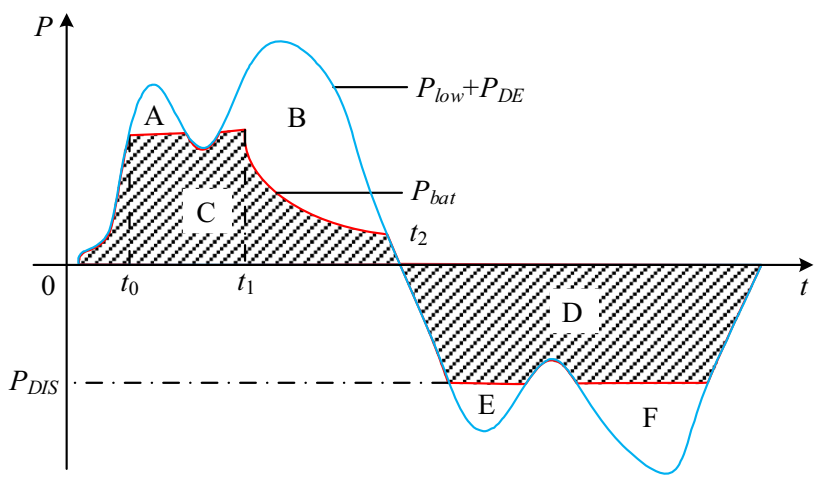

Fig. 6 Power sharing of battery unit 


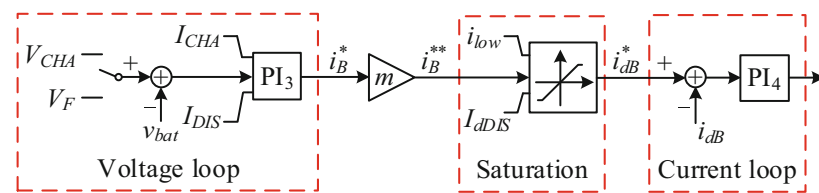

Fig. 7 Control of battery VSC

$i_{d B}^{*}=\min \left\{m I_{C H A}, i_{\text {low }}\right\}$

In contrast, if the battery stays in stages 2 or 3 of the charge process, $\mathrm{PI}_{3}$ generates a positive value between 0 and $I_{C H A}$ and $i_{d B}^{*}$ is derived as (9).

$i_{d B}^{*}=\min \left\{i_{B}^{* *}, i_{\text {low }}\right\}$

If $P_{\text {low }}+P_{D E}<0$, then $V_{C H A}>v_{b a t}$ is also satisfied and $\mathrm{PI}_{3}$ outputs its maximum value of $I_{C H A}$. Through the dynamic saturation block, the reference current $i_{d B}^{*}$ is derived as (10), where $i_{\text {low }}$ is negative.

$i_{d B}^{*}=\max \left\{I_{d D I S}, i_{\text {low }}\right\}$

From the above analysis, C and D in Fig. 6 can be extracted and compensated by the battery through local control of the VSC without requiring SOC estimations. Areas A, B, E, and F remain for further compensation.

The reactive power $Q_{\text {low }}$ should be fully compensated by the battery VSC. To accomplish this, $Q_{\text {low }}$ is directly transformed to $i_{q B}^{*}$ and assigned to the VSC controller as the reactive current set-point.

\subsection{Local control of flexible load}

The flexible load is located on the left side of the main $\mathrm{AC}$ bus with the diesel generator set. It is equipped to dump the power surplus from $P_{\text {low }}$ and from the diesel generator. Through local metering of point $C, \boldsymbol{v}_{C}$ and $\boldsymbol{i}_{C}$ are detected. The remaining slow-varying power from $P_{\text {low }}$ is calculated and defined as $P_{\text {gap }}$. With consideration for the diesel minimum power production limit $P_{D E}$, the total power that needs to be dumped is determined by (11).

$P_{\text {flex }}= \begin{cases}P_{\text {gap }}+P_{D E} & P_{\text {gap }}+P_{D E} \geq 0 \\ 0 & P_{\text {gap }}+P_{D E}<0\end{cases}$

$P_{D E}$ is a positive constant, which is within $30 \%-40 \%$ of the diesel capacity, and if $P_{g a p}+P_{D E}>0$ (A and B in Fig. 6), the flexible load will dissipate all power. Otherwise if $P_{g a p}+P_{D E}<0$, the flexible load is shut down.

Flexible load can be heat production, hydrogen production, or sea water desalination systems for example. In this work, a VSC interfaced controlled-current source (CCS) is employed as a general flexible load. The VSC is regulated as a rectifier and the DC side voltage is maintained at $V_{\text {flex }}$ through another voltage control loop, with $P_{\text {flex }} / V_{\text {flex }}$ sent to the CCS as the source signal.

\subsection{Local control of diesel generator set}

The diesel generator set works as the grid-forming unit (master unit) of the microgrid to support the main AC bus. The actuator is modeled using a third-order transfer function, and $T_{4}, T_{5}, T_{6}$ are its coefficients. Then a transport delay unit models the diesel engine with a time constant $T_{D}$. The diesel generator set is equipped with a constant speed governor, which ensures constant frequency of the output voltages. The governor is a second-order transfer function with three coefficients $T_{1}, T_{2}$, and $T_{3}$. Furthermore, the generator set is equipped with a static exciter, which ensures constant amplitude of the output voltages. The exciter is modeled using another transfer function with two confidents $T_{7}$ and $T_{8}$. The control scheme is schematically displayed in Fig. 8, where two feedback control loops ( $\omega$ in p.u. and $E$ in p.u.) are included.

The diesel generator set is an AC voltage source and its power output is regulated indirectly without communications. If $P_{\text {low }}>0$, the diesel generator set produces $P_{D E}$ and this power is totally dumped by the flexible load. If $P_{\text {low }}<0$ and is within the scope of $P_{D E}$ (a net load exists), the diesel generator set provides $P_{D E}$, one part is used to satisfy the load demand and any remaining power is dumped. However, if $P_{\text {low }}<0$ and exceeds the range of $P_{D E}$, the diesel generator set only outputs $P_{D E}$, and the demand gap is filled by the battery. If the battery is functioning at its maximum capability and more power is needed, the diesel generator set will supply the increment automatically (E and F in Fig. 6).

\section{Results and verifications}

A simulation model of an islanded AC microgrid with the same structure as Fig. 1 is built, which consists of a diesel generator set, three feeders (wind turbine, linear load, and converter load), an ultra-capacitor unit, a battery unit, and a flexible load. As to the line impedances $Z_{1}-Z_{5}$, each has a resistance of $0.092 \Omega$ and an inductance of 125 $\mu \mathrm{H}$, which simulate a $50 \mathrm{~m}$ transmission line (line diameter is about $15 \mathrm{~mm}$ ). The nominal AC bus voltage is $380 \mathrm{~V} / 50$

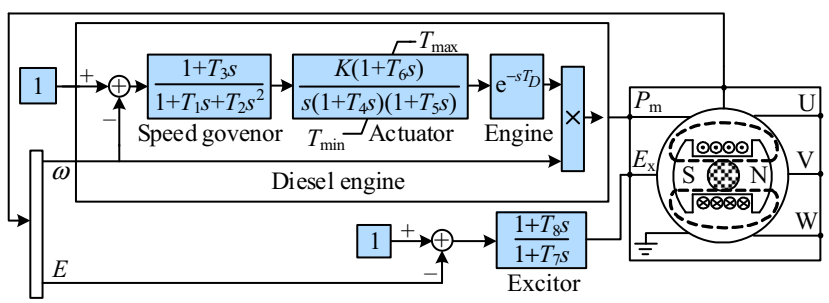

Fig. 8 Schematic of diesel generator set 
$\mathrm{Hz}$ (line-to-line) and the capacity of each unit is listed in Table 1.

In simulation, the diesel generator set is started first to build and dominate the main $\mathrm{AC}$ bus and then the ultracapacitor unit, the battery unit, and the flexible load unit are activated at $1 \mathrm{~s}, 2 \mathrm{~s}$ and $3 \mathrm{~s}$, respectively. The linear load is imposed at $5 \mathrm{~s}$ and the wind turbine is switched on at $10 \mathrm{~s}$, which is a step-up power increase to simulate gusting winds. Then the heavy converter load is imposed at $20 \mathrm{~s}$. With these designed scenarios, the power detecting and sharing process of each unit can be presented comprehensively and incisively.

Active power variations under the defined conditions are displayed in Fig. 9. The power generation of the wind turbine $P_{g e n}$, linear load $P_{R L}$, converter load $P_{c o v}$, and the total active net power $P_{\text {net }}$ are presented.

Under the power variation conditions, the performance of the microgrid system is verified. Figure 10a shows the frequency of the system, which is maintained at $50 \mathrm{~Hz}$, Fig. 10b shows the voltage amplitude of the diesel generator output, and it is also maintained constant at $311 \mathrm{~V}$, and Fig. 10c shows the waveforms of the grid voltage $v_{\text {grid }}$ when $80 \mathrm{~kW}$ active power at $10 \mathrm{~s}$ is added by the wind turbines, which only causes some slight distortions.

The decentralized control scheme without communications is verified and the active power sharing results of each unit are provided in Fig. 11. Figure 11b shows the fastvarying power that is extracted and suppressed by the ultracapacitor unit. The ultra-capacitor is activated at every power perturbation. It should be noted that for this complex simulation model, we choose $T=2 \mathrm{~s}$ for $\omega_{b}$ of the LPF to reduce the computational burden imposed by the simulation.

The slow-varying power sharing results are displayed in Fig. 11c-e. As described previously, the diesel generator set is started first and no power is produced until the battery is activated at $2 \mathrm{~s}$. Then the diesel generator set generates power $P_{D E}$ to charge the battery. When the linear load is imposed at $5 \mathrm{~s}, P_{D E}$ is maintained at constant level and the battery provides power for any shortage. When the $80 \mathrm{~kW}$ renewable power source joins the microgrid at $10 \mathrm{~s}$, the

Table 1 Capacity allocation of each unit

\begin{tabular}{ll}
\hline Unit & Value \\
\hline Wind turbine & $80 \mathrm{~kW}$ \\
Linear load & $40 \mathrm{~kW} / 10 \mathrm{kvar}$ \\
Converter load & $100 \mathrm{~kW}$ \\
Diesel generator set & $100 \mathrm{kVA}$ \\
Ultra-capacitor & $750 \mathrm{~V} / 6 \mathrm{~F}$ \\
Battery & $700 \mathrm{~V} / 180 \mathrm{Ah}$ \\
\hline
\end{tabular}

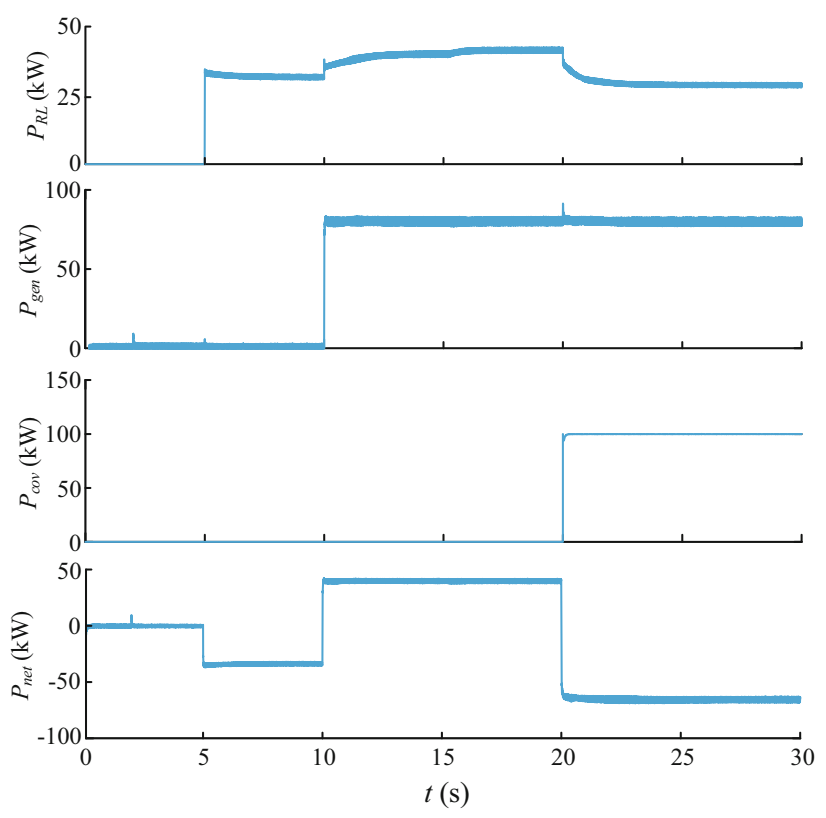

Fig. 9 Designed power variation scenarios

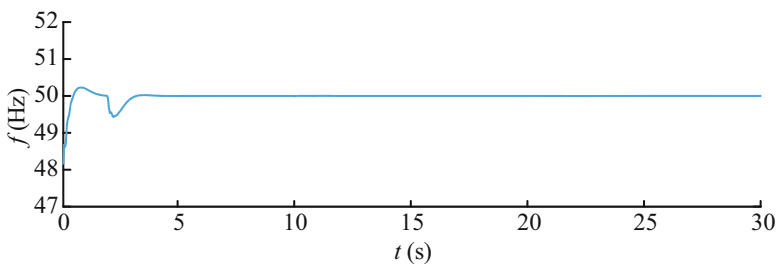

(a)

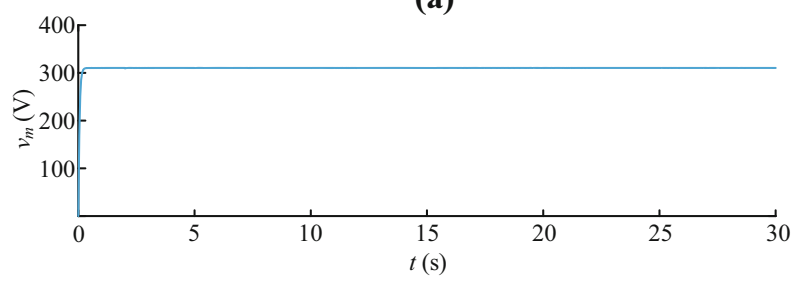

(b)

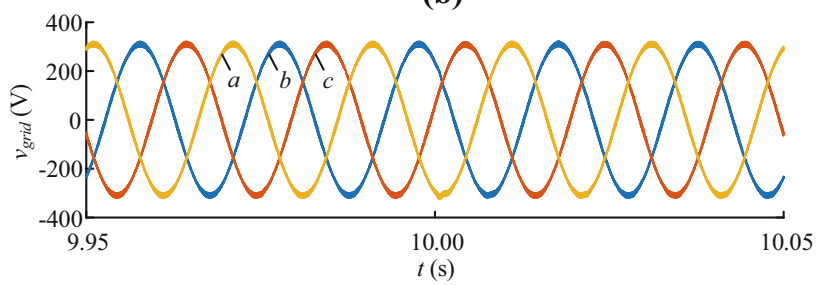

(c)

Fig. 10 Performance of microgrid system frequency $f$, voltage amplitude of diesel generator set $v_{m}$, and voltage waveforms at $10 \mathrm{~s}$

diesel set continues to work at $30 \%$ load-rate and the battery returns to a charging state. Stages 1 and 2 of the profiles in Figs. 5 and 6 are clearly demonstrated and verified. Any excess power is dumped by the flexible load. At $20 \mathrm{~s}$, a heavy converter load is imposed, which means that user demand has increased. Then the flexible load is 


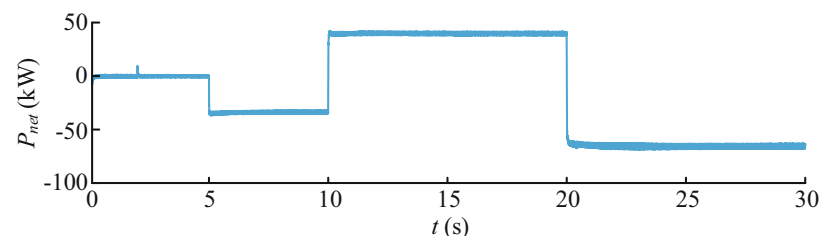

(a)

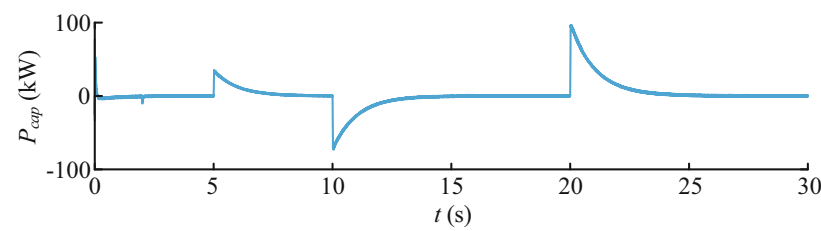

(b)

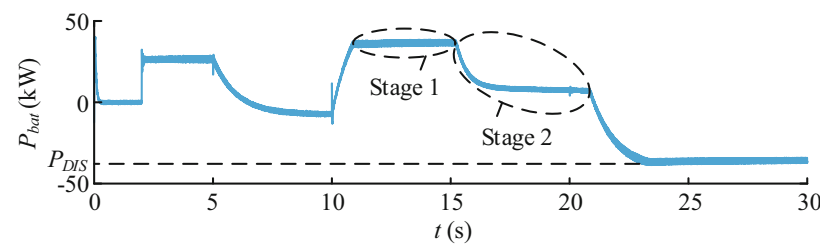

(c)

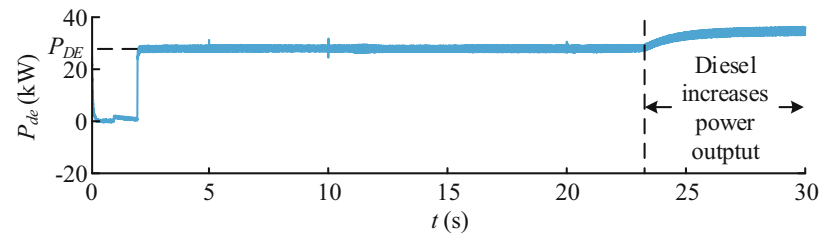

(d)

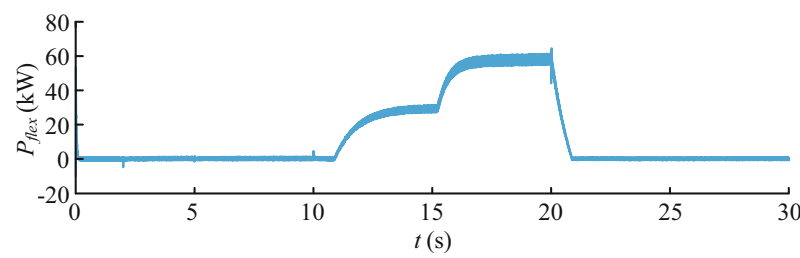

(e)

Fig. 11 Active power sharing of each unit with decentralized control schemes

shut down accordingly and the battery begins to discharge, supplying power up to its maximum capability, $P_{D I S}$. Once $P_{D I S}$ is reached, the diesel generator set supplies the power increment needed to satisfy user demand.

With the proposed system deployment principle and the decentralized control scheme, active power requirements of the microgrid can be detected and power is shared locally and independently.

As to the reactive power, approximately $10 \mathrm{kvar}$ is imposed at $5 \mathrm{~s}$ (the $R$ and $L$ linear loads). As in Fig. 12a, the reactive power $Q_{n e t}$ fluctuates during operation mainly because of the terminal voltage change of the linear loads under different conditions. The fast-varying reactive power $Q_{\text {high }}$ is suppressed by the ultra-capacitor unit through the same power calculation technique as $P_{\text {high }}$, and is displayed in Fig. 12b, where $Q_{\text {cap }}$ represents fast-varying power suppression of the ultra-capacitor. The remaining slow-

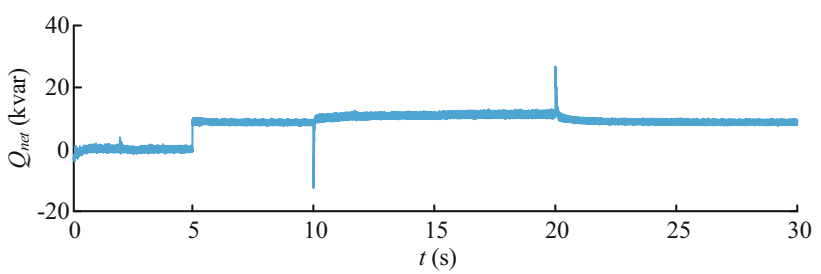

(a)

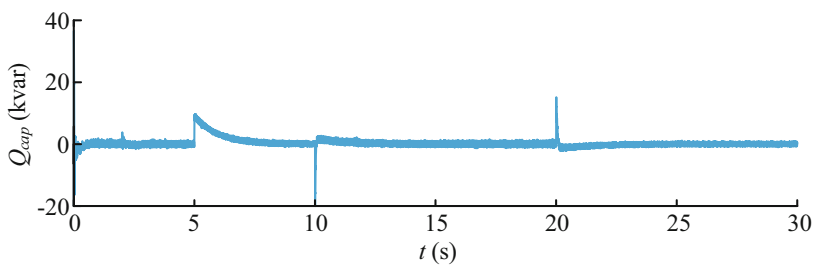

(b)

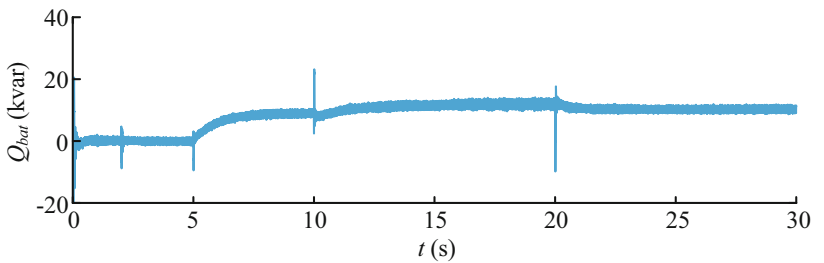

(c)

Fig. 12 Reactive power sharing of each unit under decentralized control schemes

varying reactive power $Q_{\text {low }}$ is compensated by the battery unit totally, as displayed in Fig. 12c, where $Q_{\text {bat }}$ represents slow-varying power sharing of the battery.

The ultra-capacitor is used to suppress fast-varying active power variations, and its terminal voltage can be out of balance over long-term operation owing to irregular power variation and self-discharge. As illustrated in Section 3, a rebalance algorithm is designed. The verification results are displayed in Fig. 13.

The rated voltage is $700 \mathrm{~V}$ and the tolerable operation range is defined as $\left[V_{\text {low }}, V_{u p}\right]=[695 \mathrm{~V}, 705 \mathrm{~V}]$ in this simulation model. The rebalance results are presented in Fig. 13. Without and with the rebalance algorithm, the ultra-capacitor voltage behaves as shown in Fig. 13a, b, respectively. With the proposed approach, the ultra-capacitor voltage can always be rebalanced to the rated value and the power component $P_{\text {AC }}$ needed for voltage rebalance under the defined conditions is shown in Fig. 13c.

\section{Conclusion}

Microgrid control always relies on communication facilities to achieve power balance and economic targets. This work proposes an innovative system deployment principle based on a decentralized power flow control scheme without communication facilities for a master/slave type islanded AC microgrid. This difference will benefit 


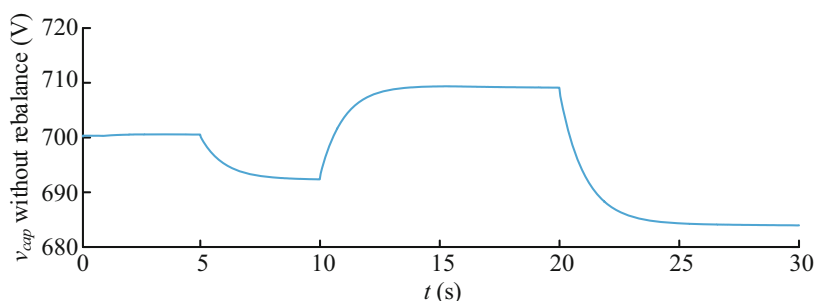

(a)

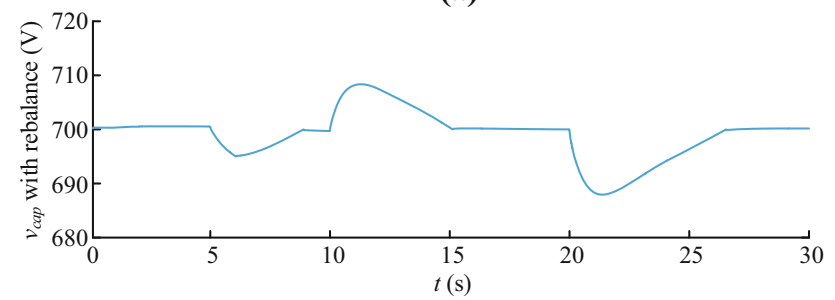

(b)

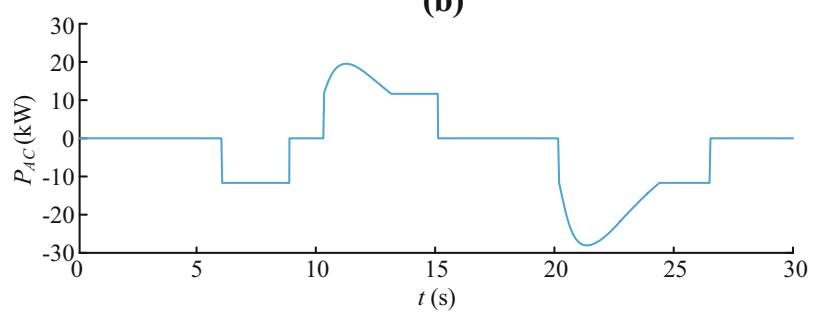

(c)

Fig. 13 Rebalance of ultra-capacitor

the flexibility and plug-and-play of a microgrid. A smallscale AC microgrid with diesel, wind, hybrid storage, critical loads, and flexible loads is investigated. Without using any communications among units, the net power, including active and reactive power, are detected and compensated locally and independently considering the diesel set's low load-rate operation, battery charging, and ultra-capacitor rebalance issues. Finally, simulation results are provided to verify the validity of the proposed deployment principle based on decentralized control.

Open Access This article is distributed under the terms of the Creative Commons Attribution 4.0 International License (http:// creativecommons.org/licenses/by/4.0/), which permits unrestricted use, distribution, and reproduction in any medium, provided you give appropriate credit to the original author(s) and the source, provide a link to the Creative Commons license, and indicate if changes were made.

\section{References}

[1] Lasseter RH, Paigi P (2004) Microgrid: a conceptual solution. In: Proceedings of 2004 IEEE 35th annual power electronics specialists conference, Aachen, Germany, 20-25 June 2004, pp 4285-4290

[2] Ribeiro LADS, Saavedra OR, Lima SLD et al (2011) Isolated micro-grids with renewable hybrid generation: the case of Lençóis island. IEEE Trans Sustain Energy 2(1):1-11
[3] Shi H, Zhuo F, Yi H et al (2015) A novel real-time voltage and frequency compensation strategy for photovoltaic-based microgrid. IEEE Trans Ind Electron 62(6):3545-3556

[4] Zhu Y, Zhuo F, Wang F et al (2015) A wireless load sharing strategy for islanded microgrid based on feeder current sensing. IEEE Trans Power Electron 30(12):6706-6719

[5] Chen D, Xu L (2012) Autonomous DC voltage control of a DC microgrid with multiple slack terminals. IEEE Trans Power Syst 27(4):1897-1905

[6] Liu X, Shahidehpour M, Li Z et al (2017) Microgrids for enhancing the power grid resilience in extreme conditions. IEEE Trans Smart Grid 8(2):589-597

[7] Han H, Hou X, Yang J et al (2016) Review of power sharing control strategies for islanding operation of AC microgrids. IEEE Trans Smart Grid 7(1):200-215

[8] Hatziargyriou N (2014) Microgrids: architectures and control. Wiley-IEEE Press, New Jersey, p 344

[9] Tian P, Xiao X, Wang K et al (2016) A hierarchical energy management system based on hierarchical optimization for microgrid community economic operation. IEEE Trans Smart Grid 7(5):2230-2241

[10] Han Y, Shen P, Zhao X (2017) Control strategies for islanded microgrid using enhanced hierarchical control structure with multiple current-loop damping schemes. IEEE Trans Smart Grid 8(3):1139-1153

[11] Brandao DI, Caldognetto T, Marafão FP et al (2017) Centralized control of distributed single-phase inverters arbitrarily connected to three-phase four-wire microgrids. IEEE Trans Smart Grid 8(1):437-446

[12] Elrayyah A, Cingoz F, Sozer Y (2017) Smart loads management using droop-based control in integrated microgrid systems. IEEE J Emerg Sel Top Power Electron 5(3):1142-1153

[13] Malik SM, Ai X, Sun Y (2017) Voltage and frequency control strategies of hybrid AC/DC microgrid: a review. IET Gener Transm Distrib 11(2):303-313

[14] Karimi Y, Oraee H, Guerrero JM (2017) Decentralized method for load sharing and power management in a hybrid single/ three-phase-islanded microgrid consisting of hybrid source PV/battery units. IEEE Trans Power Electron 32(8):6135-6144

[15] Ahumada C, Cárdenas R, Sáez D et al (2016) Secondary control strategies for frequency restoration in islanded microgrids with consideration of communication delays. IEEE Trans Smart Grid 7(3):1430-1441

[16] Bevrani H, Feizi MR, Ataee S (2016) Robust frequency control in an islanded microgrid: $H \infty$ and $\mu$-synthesis approaches. IEEE Trans Smart Grid 7(2):706-717

[17] Chen X, Hou Y, Hui SYR (2017) Distributed control of multiple electric springs for voltage control in microgrid. IEEE Trans Smart Grid 8(3):1350-1359

[18] Shamsi P, Xie H, Longe A et al (2016) Economic dispatch for an agent-based community microgrid. IEEE Trans Smart Grid 7(5):2317-2324

[19] Hamad AA, Azzouz MA, El-Saadany EF (2016) Multiagent supervisory control for power management in DC microgrids. IEEE Trans Smart Grid 7(2):1057-1068

[20] Li Q, Chen F, Chen M et al (2016) Agent-based decentralized control method for islanded microgrids. IEEE Trans Smart Grid 7(2):637-649

[21] Pelland S, Turcotte D, Colgate G et al (2012) Nemiah valley photovoltaic-diesel mini-grid: system performance and fuel saving based on one year of monitored data. IEEE Trans Sustain Energy 3(1):167-175

[22] Vidyanandan KV, Senroy N (2016) Frequency regulation in a wind-diesel powered microgrid using flywheels and fuel cells. IET Gener Transm Distrib 10(3):780-788 
[23] Christen T, Carlen M (2000) Theory of Ragone plots. J Power Sources 91:210-216

[24] Sathishkumar R, Kollimalla SK, Mishra MK (2012) Dynamic energy management of micro grids using battery super capacitor combined storage. In: Proceedings of 2012 annual IEEE India conference (INDICON), Kochi, India, 7-9 December 2012, pp 1078-1083

[25] Tankari MA, Camara MB, Dakyo B et al (2013) Use of ultracapacitors and batteries for efficient energy management in wind-diesel hybrid system. IEEE Trans Sustain Energy $4(2): 414-424$
Baoquan LIU received his B.S. degree in electrical engineering from Harbin Institute of Technology, Harbin, China, in 2009. He received his M.S. degree and Ph.D. degree from Xi' an Jiaotong University Xi'an, China, in 2012 and 2015, respectively. He is currently an associate professor with the School of Electrical and Information Engineering in Shaanxi University of Science and Technology. His research interests include system design and power flow control of DC and AC microgrids using hybrid energy storage. 